\title{
Spectral collapse in ensembles of meta-molecules
}

\author{
V. A. Fedotov,${ }^{1, *}$ N. Papasimakis,${ }^{1}$ E. Plum,${ }^{1}$ A. Bitzer, ${ }^{2}$ M. Walther, ${ }^{2}$ P. Kuo, ${ }^{3}$ D. P. Tsai, ${ }^{4}$ and N. I. Zheludev ${ }^{1, \dagger}$ \\ ${ }^{1}$ Optoelectronics Research Centre, University of Southampton, SO17 1BJ, UK \\ ${ }^{2}$ Department of Molecular and Optical Physics, University of Freiburg, D-79104, Germany \\ ${ }^{3}$ Institute of Physics, Academia Sinica, Taipei, 11529, Taiwan \\ ${ }^{4}$ Department of Physics, National Taiwan University, Taipei 10617, Taiwan
}

(Dated: August 5, 2009)

\begin{abstract}
We report on a new collective phenomenon in metamaterials: spectral line collapse with increasing number of the unit cell resonators (meta-molecules). Resembling the behaviour of exotic states of matter, such as Bose-Einstein condensates of excitons and magnons, this new effect is linked to the suppression of radiation losses in periodic arrays. We demonstrate experimentally spectral line collapse at microwave, terahertz and optical frequencies. It emerges as a universal and truly scalable effect underpinned by classical electromagnetic interactions between the excited meta-molecules.
\end{abstract}

The burgeoning field of metamaterials provides unique opportunities to engineer the electromagnetic properties of artificial media and achieve exotic functionalities, such as negative refraction [1] and cloaking [2]. Similarly to natural crystals, which are created by arranging individual atoms and molecules in a regular grid, periodic ensembles of subwavelength electromagnetic resonators present an effective medium to an incident with properties not available in natural materials. Here, we study the dependence of the metamaterial properties on the number of meta-molecules in the microwave, $\mathrm{THz}$ and optical domain, and demonstrate a new collective phenomenon in metamaterials: in contrast to solid state crystals, where bulk arrangements result in broadening of the individual element spectral line, leading eventually to the formation of absorption bands, regular ensembles of meta-molecules can exhibit the opposite effect, i.e. spectral line collapse.

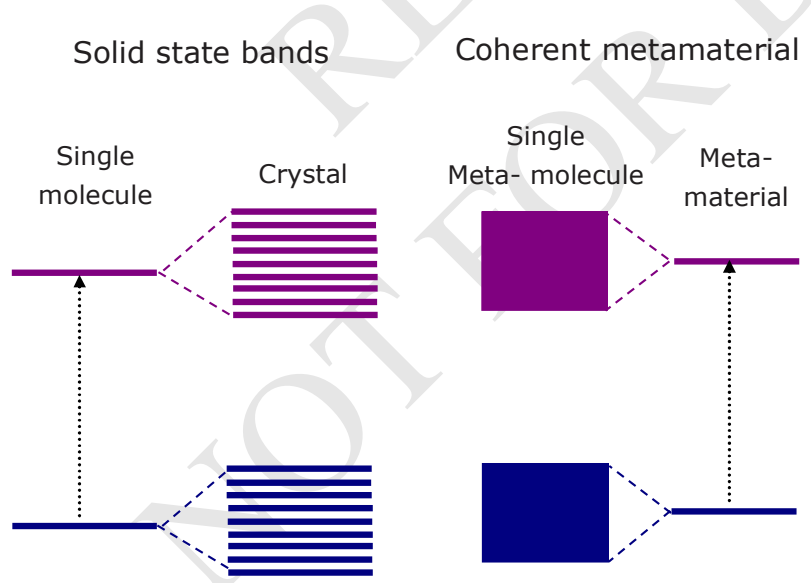

FIG. 1: (Colour online) Resonant absorption lines of atoms form a broader absorption band in solids. In coherent metamaterials we see the opposite trend: resonant lines of large arrays are much narrower than lines of individual metamolecules.

The reported phenomenon is characteristic to a novel class of artificial media, which we call "coherent" meta- materials [3] and are characterized by very strong interactions between the electromagnetically excited metamolecules that provide for a low rate of energy loss due to scattering and lead to a high-quality resonant response. An example of a coherent metamaterial is an array of ASRs, where the meta-molecular excitation corresponds to an oscillating magnetic dipole perpendicular to the plane of the array that does not interact directly with the magnetic field of the incident wave, thus creating a nearly thermodynamically isolated ensemble of strongly interacting coherent "molecules" with interesting physical properties. To illustrate this behavior we present a comparison with an "incoherent" metamaterial: a twodimensional array formed by pairs of concentric conducting rings that also supports a high-quality resonant response. In this case, however, the response of the array is a sum of the individual meta-molecule responses, rather than a collective property.

The coherent microwave metamaterial was manufactured as a regular planar array of asymmetric split rings (ASR) etched from a $35 \mu \mathrm{m}$ thick copper layer on a $1.6 \mathrm{~mm}$ thick FR4 substrate. The diameter of the ASR was $6 \mathrm{~mm}$ with a line width of $0.4 \mathrm{~mm}$ and was split in two segments corresponding to $140^{\circ}$ and $160^{\circ}$ arcs. The unit cell of $7.5 \times 7.5 \mathrm{~mm}^{2}$ rendered the arrays nondiffracting at normal incidence for frequencies of up to $40 \mathrm{GHz}$. In the incoherent metamaterial ASRs were replaced with pairs of concentric rings. The inner and outer rings had the diameters of correspondingly $4.50 \mathrm{~mm}$ and $5.45 \mathrm{~mm}$, and were both $0.2 \mathrm{~mm}$ wide. The transmission measurements were performed in a microwave anechoic chamber at normal incidence using broadband linearly polarized horn antennas equipped with collimating lenses and a vector network analyzer.

Transmission spectra of large coherent and incoherent arrays show similar resonant features in the form of a broad stop-band split by a narrower Fano-like transmission peak (see Fig. 2). For the array of ASRs the transmission resonance occurs at around $11 \mathrm{GHz}$ and is associated with the excitation of anti-symmetric currents oscillating in the opposite arcs of each split ring (see in- 


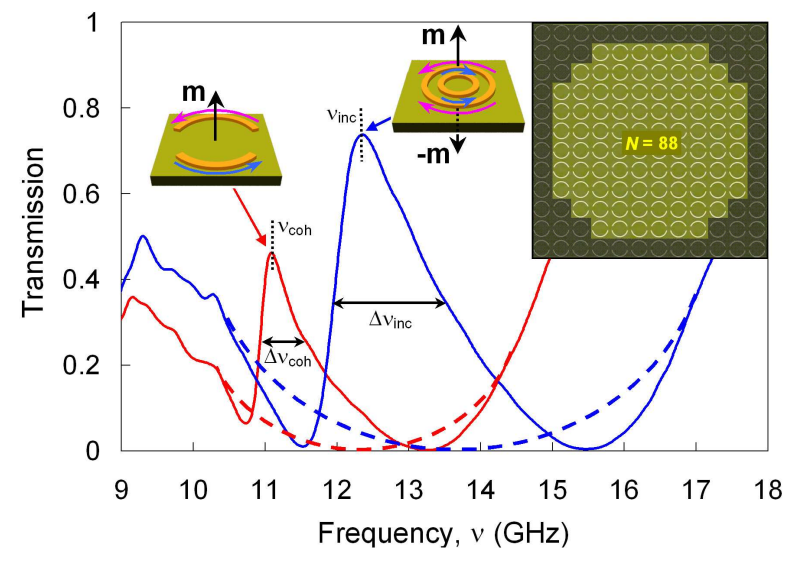

FIG. 2: (Colour online) Transmission spectra feature trappedmode resonances of the coherent (asymmetric split rings, solid red curve, $\nu_{c o h}$ ) and incoherent (concentric rings, solid blue curve, $\nu_{\text {inc }}$ ) metamaterial arrays of 88 meta-molecules. Dashed curves indicate transmission stop bands of the metamaterials in the absence of trapped-mode excitations. The top right inset shows a sample of 88 meta-molecules (unit cells) of the coherent array exposed through a metal mask (represented by the darker area). Other insets show the trapped current modes excited in the unit cells of the coherent (left) and incoherent (right) metamaterials and the corresponding induced magnetic dipole moments $m$.

set to Fig. 2). Such a current mode, known as a trapped or closed mode, yields magnetic dipole moments oscillating synchronously (coherently) in all meta-molecules along the direction normal to the plane of the array. The induced magnetic dipoles interact strongly with one another, while their interaction with the perpendicularly oriented magnetic field of the incident electromagnetic wave is forbidden [4]. For the double-ring metamaterial a similar narrow resonance at $13 \mathrm{GHz}$ corresponds to oscillations of oppositely directed currents in the inner and outer rings, as shown in the inset to Fig. 2. Although such current configurations give rise to magnetic moments, the latter cancel one another and the total magnetic moment of the meta-molecule and thus interactions between meta-molecules are negligible [5].

Figure 3 shows the dependencies of the transmission resonance quality factors, $Q=\nu / \Delta \nu$, on the total number of meta-molecules, $N$, that form the arrays. In the experiment, the number of meta-molecules exposed to the incident wave was controlled by placing metal masks of different sizes on a large metamaterial array, as illustrated in the inset to Fig. 2. These masks screened the peripheral parts of the array, leaving the central part of the array exposed to electromagnetic radiation. The shape of the masks was close to circular with a step-like profile of the opening, which ensured that every unit cell of the array was either fully screened or exposed. These measurements were performed with 22 different masks exposing arrays with a total number of unit cells in the range from 32 to about 700. Experiments with larger arrays were not practical and unnecessary as already for $N \simeq 700$ the quality factor saturated. Experiments with smaller arrays were not feasible as for $N<32$ diffraction takes its toll on the accuracy of the data. In the measured range of $N$ the experimental data clearly indicate that the Q-factor of the coherent metamaterial strongly depends on the size of the illuminated area, i.e., the total number of meta-molecules engaged in the interaction with the incident wave. Indeed, the Q-factor, which measures about 10.5 for the smallest array $(N=32)$, can be seen to increase by almost $70 \%$ reaching 17.5 for the fullsized array with $N=688$. In contrast, the Q-factor of the incoherent metamaterial appears to be practically independent of the number of exposed unit cells and remains at around $Q=9$.

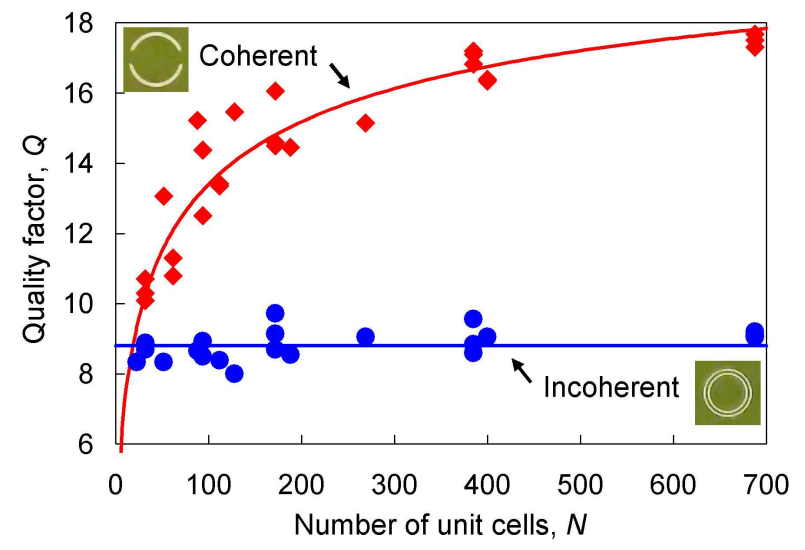

FIG. 3: (Colour online) Graphs show Q-factors as a function of the total number of meta-molecules. Experimentally measured data are represented by points for both coherent (diamonds) and incoherent (circles) metamaterials. Solid lines present theoretical fits to the data.

To illustrate further the dependence of the resonant response on the size of the coherent metamaterial, we studied the spatial distribution of the magnetic field in a single split-ring meta-molecule and a meta-molecule that is a part of a regular array containing $N=400$ unit cells. For this we employed a $\mathrm{THz}$ near-field imaging technique with sub-wavelength resolution described in [6]. The technique enabled accurate mapping of orthogonal components of the electrical field $\vec{E}$ in the plane of the unit cell (as illustrated in the inset to Fig. 4a), which was used to calculate the component of the magnetic field $\vec{H}$ normal to the plane of the structure via the Maxwell equation $\vec{\nabla} \times \vec{E}=-\partial \vec{B} / \partial t$.

In our experiment we observed that at resonance conditions the spatial distribution of the magnetic field was radically different for a split-ring placed in the array and an isolated split-ring. For a ring in the array the magnetic field penetrates the unit cell in the same direction everywhere within the area enclosed by the ring: the 
(a)
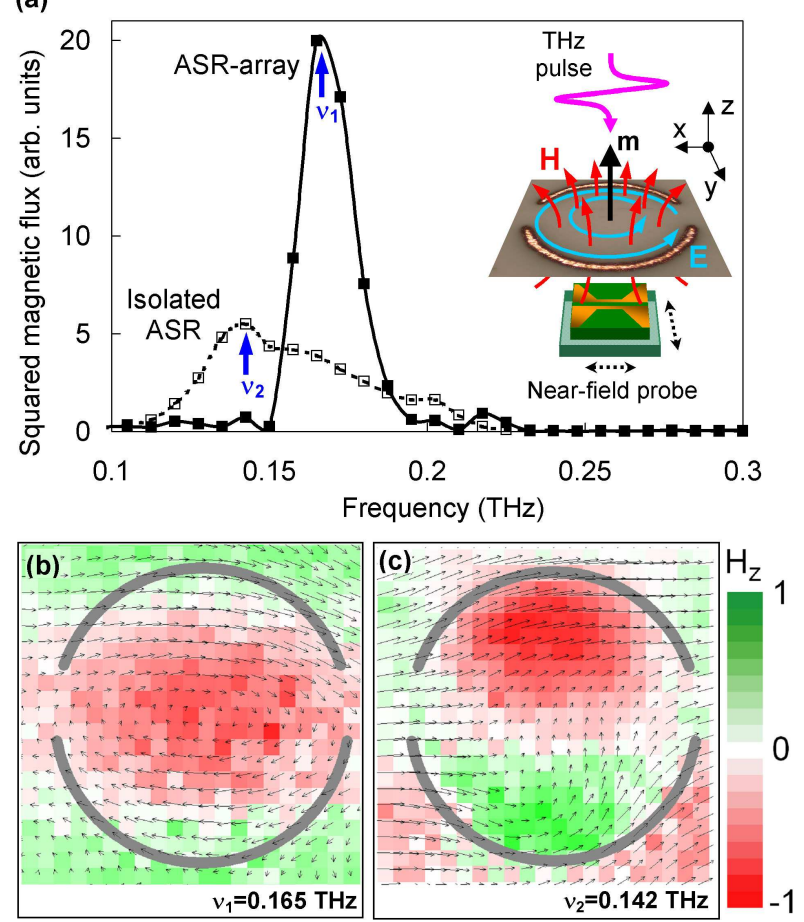

FIG. 4: (Colour online) Panel (a) shows the spectral dependence of the squared total magnetic flux amplitude through an ASR for the cases of one isolated ring (empty squares, weak magnetic response) and a ring located in the middle of a $20 \times 20$ array (filled squares, strong magnetic response). Panels (b) and (c) present snap-shot maps of the instantaneous component of the magnetic field normal to the plane of the ring (colour coded) and the in-plane component of the electric field (arrows) at resonance frequencies $\nu_{1}$ and $\nu_{2}$ correspondingly (see also supplementary videos for temporal evolution of the fields). In order to show both fields at their maxima on the same map the magnetic and electric fields are presented with phase shift of $\pi / 4$.

total magnetic moment of the meta-molecule is at maximum indicating the excitation of strong anti-symmetric (i.e. ring) currents in opposite sections of the ring (see Fig. 4b), while the spectral dependence of the squared amplitude of the magnetic flux reveals a narrow sharp peak centered at $\nu_{1}=0.165 \mathrm{THz}$ (see Fig. 4a). For an isolated single meta-molecule the net magnetic flux is also non-zero but it is much lower than for a ring in the array: the magnetic field is oppositely directed in adjacent sections of the ring (see Fig. 4c), the total magnetic flux is small and the anti-symmetric current component is weak. As a result, the resonant feature at $\nu_{2}=0.142 \mathrm{THz}$ is much less pronounced and its Q-factor is significantly lower than for rings in the array indicating considerable damping (see Fig. 4a).

We attribute the observed size-dependent resonant response of the coherent metamaterial to the existence of strong interactions between the meta-molecules mediated by magneto-inductive surface waves [7]. It follows from symmetry arguments that magnetic dipoles coherently oscillating perpendicular to the plane of an infinite (or very large) regular array can re-radiate only in its plane, which ensures very low scattering losses and therefore a high Q-factor of the system. Electromagnetic energy in the coherent array is trapped in the form of standing magneto-inductive surface waves, which may only scatter on the edges of the array. The scattering losses grow and the Q-factor diminishes as the array becomes smaller, and in the limiting case of an isolated meta-molecule the resonance is extremely weak due to intense energy losses through magnetic dipole radiation (see also supplementary materials).

The dependence of the Q-factor on the size of the array can be qualitatively understood from the definition of the Q-factor, $Q=2 \pi \cdot E / \Delta E$, where $E$ is the energy stored in the array and $\Delta E$ is the energy dissipated by the array per cycle. Here $E$ is simply proportional to $N$, while $\Delta E=\Delta E_{m}+\Delta E_{s}$ represents contributions from two loss mechanisms. $\Delta E_{m}=\sigma_{m} \cdot N$ includes material losses, i.e., Ohmic losses in the metal and dissipation losses in the dielectric substrate, as well as radiation losses due to an electric dipole induced in each unit cell, which is controlled by the degree of asymmetry of the split ring. Losses associated with scattering of the magneto-inductive waves on the edges of the array, $\Delta E_{s}$, are proportional to the length of its perimeter and thus $\Delta E_{s}=\sigma_{s} \cdot \sqrt{N}$. Thus, the phenomenological dependence of the quality factor on the number of unit cells can be presented in the form $Q \propto N /\left(\sigma_{m} \cdot N+\sigma_{s} \sqrt{N}\right)$, which fits the experimental data very well (solid red line in Fig. 3). In comparison, in the case of the incoherent array of concentric pairs of rings, the total induced magnetic moment perpendicular to the plane of the array is small, hence magnetic dipole interactions and scattering of magnetoinductive waves are negligible $\left(\sigma_{m} \cdot N \gg \sigma_{s} \sqrt{N}\right)$. The second term in the denominator can be disregarded making the Q-factor independent of the size of the array, which is in complete agreement with our experimental observations (solid blue line in Fig. 3).

The dependence of the resonant response on the array's size results from truly classical interactions between the excited states of the meta-molecules and therefore shall be universal and truly scalable with wavelength. Since the characteristic size of the array at which substantial broadening of the resonance is seen is given by $N=\left(\sigma_{s} / \sigma_{m}\right)^{2}$, with increase of Joule losses the size dependent effect will be seen in smaller arrays, i.e., in an array with higher Joule losses the coherent state will be formed by a smaller number of meta-molecules.

This is exactly what we observed in our experiments in the optical part of the spectrum, where Joule losses are dominant. Here we studied transmission of square asymmetric split ring slit arrays of different sizes scaled down 15,000 times relative to the microwave sample. The ring slits (complimentary to split-rings) were cut from 
a $55 \mathrm{~nm}$ thick gold film supported by a $500 \mu \mathrm{m}$ silica substrate using focused ion beam milling. The transmission measurements were performed using a microscopebased spectrophotometer by CRAIC Technologies. Because of the negative configuration the size of the illuminated area in transmission was naturally controlled by the apertures of the arrays, while unstructured square windows of the same size were used as reference. We studied arrays of five different sizes containing from 16 to 144 meta-molecules (see Fig. 5a - 5b). Such photonic metamaterials exhibited a trapped-mode resonance at around $1000 \mathrm{~nm}$, which, in full accordance with Babinet's principle, was seen not as a transmission peak (as for a positive array, see Fig. 2), but as a dip in transmission for the largest array of $12 \times 12$ unit cells (see inset to Fig. 5c). For smaller arrays the resonance gradually became broader and shallower and, as shown in Fig. 5c, completely disappeared for $N=16$, which suggests that the most dramatic change occurs when the number of unit cells drops below 49 .

(a)

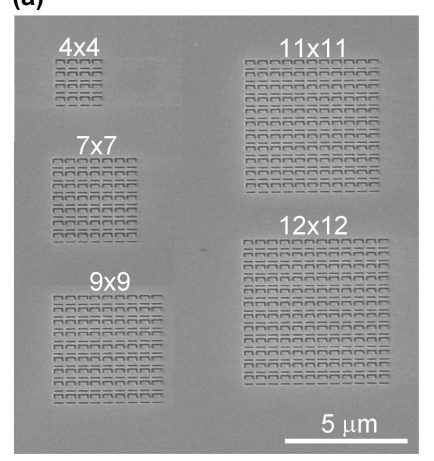

(c)

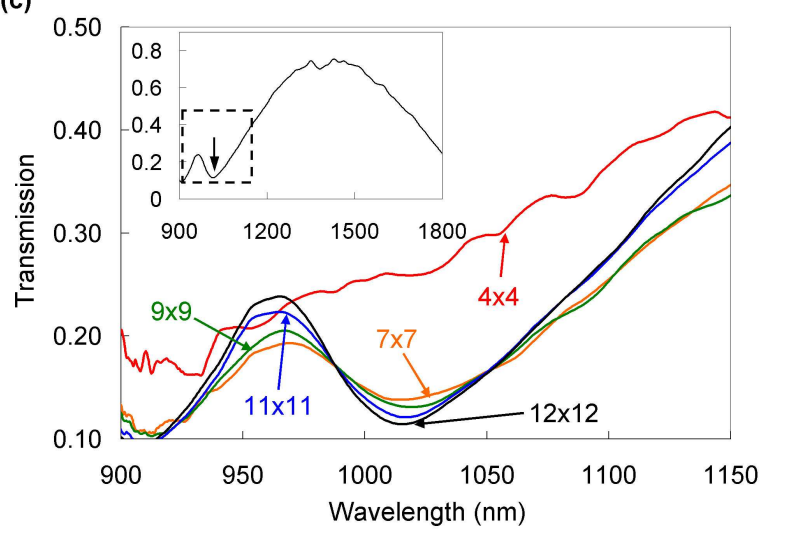

(b)

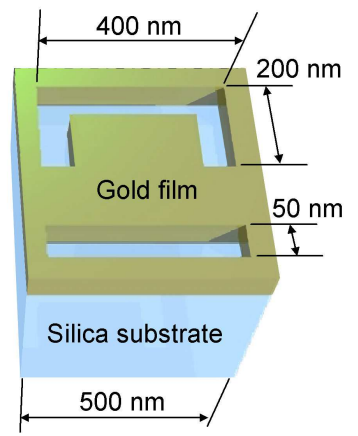

FIG. 5: (Colour online) Panel (a) shows SEM images of coherent metamaterial arrays of different sizes. Panel (b) presents a schematic of the metamaterial unit cell. Panel (c) shows transmission spectra of the metamaterial arrays in the vicinity of the trapped-mode resonance. The inset presents the transmission spectrum of the $12 \times 12$ array over a much wider wavelength range; a dashed box indicates the spectral domain that is covered by the main plot, while an arrow marks the position of the trapped-mode resonance.
The results presented above illustrate that larger arrays of coherent metamaterials exhibit narrower resonances. They also show that in samples with smaller losses more meta-molecules are engaged in forming the coherent state: the Q-factor starts reducing dramatically below about 200 meta-molecules in the microwave array, while in the lossy optical case we saw a significant reduction of Q-factor below 49 meta-molecules. We argue that the number of molecules forming the coherent response will increase as Joule and dielectric losses are compensated. This is relevant to the recent observation of loss compensation in a coherent photonic metamaterial [8], which paves the way to an intriguing opportunity of creating a lasing spaser, a coherent source of optical radiation fuelled by coherent plasmonic oscillations in individual metamaterial resonators of a coherent array. In the latter case the gain substrate supporting the rings would be the source of energy [9].

In conclusion, we would like to point out that the spectral collapse of the metamaterial resonance with increasing number of meta-molecules has intriguing phenomenological similarity to the narrowing of luminescence/scattering spectra upon formation of the BoseEinstein condensates (BEC) of exciton and magnon in solids [10-12]. The latter is observed when concentration of the bosonic quasi-particles in equilibrium exceeds a certain critical value and most of the particles are statistically forced to occupy the ground level forming a stable coherent eigenstate. In the case of the excitons, for example, the narrow emission line of the condensate corresponds to the binding energy of the quasi-particles and results from their recombination in the condensate. The analogy between these two phenomena becomes apparent since the ground state of the metamaterial system is achieved through de-excitation of the low scattering (i.e. trapped) electromagnetic mode of interacting metamolecules. Moreover, as in the BEC, the collapse of the resonant line is most rapid when the number of metamolecules reaches a certain critical value $\left(N_{c} \approx 100\right.$ in the case of the microwave metamaterial).

* Electronic address: vaf@orc.soton.ac.uk

$\dagger$ URL: www.nanophotonics.org.uk/niz

[1] D. R. Smith and J. B. P. andM. C. K. Wiltshire, Science 305, 788 (2004).

[2] D. Schurig, J. J. Mock, B. J. Justice, S. A. Cummer, J. B. Pendry, A. F. Starr, and D. R. Smith, Science 314, 977 (2006).

[3] N. Papasimakis, V. A. Fedotov, Y. H. Fu, D. P. Tsai, and N. I. Zheludev, Phys. Rev. B 80, 041102(R) (2009).

[4] V. A. Fedotov, M. Rose, S. L. Prosvirnin, N. Papasimakis, and N. I. Zheludev, Phys. Rev. Lett. 99, 147401 (2007).

[5] N. Papasimakis, Y. H. Fu, V. A. Fedotov, S. L. Prosvirnin, D. P. Tsai, and N. I. Zheludev, Appl. Phys. 
Lett. 94, 211902 (2009).

[6] A. Bitzer, H. Merbold, A. Thoman, T. Feurer, H. Helm, and M. Walther, Opt. Express 17, 3826 (2009).

[7] E. Shamonina, V. A. Kalinin, K. H. Ringhofer, and L. Solymar, J. Appl. Phys. 99, 6252 (2002).

[8] E. Plum, V. A. Fedotov, P. Kuo, D. P. Tsai, and N. I. Zheludev, Opt. Express 17, 8548 (2009).

[9] N. I. Zheludev, S. L. Prosvirnin, N. Papasimakis, and V. A. Fedotov, Nat. Photonics 2, 351 (2008).
[10] J. L. Lin and J. P. Wolfe, Phys. Rev. Lett. 71, 1222 (1993).

[11] S. O. Demokritov, V. E. Demidov, O. Dzyapko, G. A. Melkov, A. A. Serga, B. Hillebrands, and A. N. Slavin, Nature 443, 430 (2006).

[12] A. V. Chumak, G. A. Melkov, V. E. Demidov, O. Dzyapko, V. L. Safonov, and S. O. Demokritov, Phys. Rev. Lett. 102, 187205 (2009). 